\title{
Review of nano technology of Covid 19 vaccines \\ BY
}

Mohamed Ashraf Mohamed, M.A.M.,

Pharmaceutics department, Egyptian Russian University, ERU

$$
\begin{aligned}
& \text { Doi: 10.21608/asajs.2021.159448 }
\end{aligned}
$$

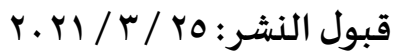

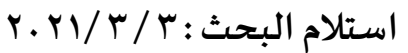

\section{Introduction :}

The global current situation, no return to pre-Covid 19 social pattern unless development of safe preventive vaccines. Fortunately, in the last few months since identity of SARS CoV-2more than two hundred academic laboratories \&companies initiated vaccine development, and many are

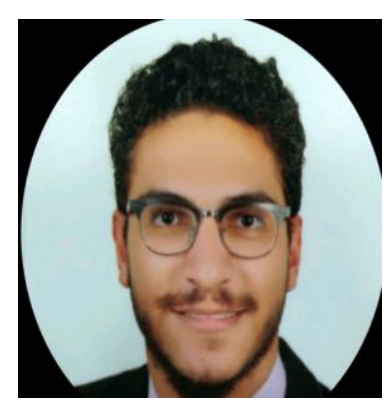
making record time in advancing to clinical trials (Pene, et.al., (2003).

\section{The COVID-19 Vaccine Candidates landscape}

According to the WHO and the Milken Institute, as of August 11, 2020, there are 202 companies and universities worldwide working on a coronavirus vaccine The vaccine types vary from well-established vaccines (e.g., inactivated and liveattenuated) to vaccines that have recently gained clinical approval (e.g., subunit) to those that have not yet made the transition into the clinic (e.g., mRNA, DNA, non-replicating viral vector, replicating viral vector) (Figure 1). To develop effective SARS-CoV-2 vaccines, a multifaceted strategic approach to vaccine development is globally pursuit. The classical vaccine such as inactivated or live-attenuated vaccines 
has advantages, such as stimulation of pattern recognition receptors and high immunogenicity.

The viruses still alive and replicable but non-toxic. However, due to the risk of live viruses, long-term surveillance is recommended for assessing the safety of the vaccine. Several inactivated virus vaccines are currently being developed against SARS-CoV-2, and the first clinical trials by Sinovac Biotech, Beijing, China, have recently started recombinant viral DNA fragments SARS-CoV using synthetic genomics. Thao, et.al, (2020).

Based on these findings, it is possible to approach a slightly more rapid generation of live-attenuated vaccines against SARS-CoV-2. Additionally, Codagenix, Farmingdale, NY, USA) Chen, et.al, 2020 is exploring vaccine candidates against SARS-CoV-2 using a "codon-optimized off" strategy for virus attenuation.

Of the 202 companies, only a select few have advanced into clinical trials; as of August 11, 2020, the WHO indicates there are 29 vaccine candidates in clinical trials (Figure 2). Of these select few vaccines, even fewer have released data of their initial safety and immunogenicity from completed Phase I and II studies. It is noteworthy that every company that has released data has reported positive results from their early Phase clinical trials, allowing advancement into wider and more broadly encompassing efficacy studies. Recombinant vaccines allow live viruses to retain some additional genes derived from pathogens through genetic manipulation, thereby translating the target protein and triggering the desired immune response. Recombinant vaccine advantages gave enough target protein expression, prolonged stability, with strong immune responses. Bull, et.al, (2019). Vaccinia virus vector-based vaccines are currently being evaluated for use in many clinical trials based on the studies that have shown that they can induce very strong 
immune responses to foreign antigens. Another advantage of the Vaccinia virus vector-based vaccine could be the availability of a large-scale manufacturing method, as in the case where Bavarian Nordic A/S produced and provided large amounts of its own smallpox vaccine IMVAMUNE ${ }^{\circledR}$ to the US government. Frey, et.al, (2013).

The broad spectrum viral affinity and infectivity in dividing and non-dividing cells has made it possible to use a wide range of adenovirus (Ad) vectors to our advantage. Among the human Ad sera identified to date, human Ad serotype 5 (Ad5), which can be easily produced at high titers, is the most widely studied gene transfer vector. However, pre-existing immunity against Ad induced in many people who have already been exposed to the Ad serotype is a disadvantage of Ad vectors. Sicot , et.al, (2011). Adeno-associated virus (AAV) is a nonpathogenic, low immunogenic, vector-enclosed, single-stranded DNA virus. AAV has both the characteristics and advantages of Ads. AAV vectors require a very efficient large-scale production method, such as the baculovirus system, which has been developed because of their low titer production efficiency. AAV is better than Ad when continuous transgene expression is required for treatment. The capsid modification vector is an alternative to overcome the low immunogenicity of AAV vectors. Mixed capsids generated from different serotypes provide effective gene transfer and tropism to host cells. Since AAV vectors often require integration with the host genome for viral gene expression, genotoxicity risk assessment must be considered when using AAV vectors. Li, et.al,(2011). ChAdOx1 nCoV-19 (AZD1222), an Ad-based recombinant vaccine developed at the University of Oxford, Oxford, UK, was found to be resistant in the Phase I/II COV001 trial, and a strong immune response to SARS-CoV-2 was generated in all 
participants (Figure 1). Almost all participants receiving AZD1222 showed a four-fold increase in the antibody neutralizing activity against the SARS-CoV-2 S protein. In addition, no serious side effects were reported with the use of AZD1222. Echevarría-Zuno, et.al,.(2009).

Inactivated vaccines are similar to the native pathogen but are replication deficient due to chemical or heat treatment. Liveattenuated vaccines are weakened forms of the virus that can replicate in a limited manner unable to cause the actual disease, and subunit vaccines confer immuno-protection using portions of the virus. Subunit vaccines are usually less immunogenic and require an adjuvant to stimulate the immune recognition of the antigens in the vaccine. Nucleic acid based vaccines can be mRNA or DNA based, and rather than directly injecting the antigen, expresses it within host cells using the genomic material.(Vartak, A. and Sucheck (2016).

Finally, viral vector vaccines have engineered genomes to encode the antigen of the target pathogen. When taken in vivo, the viral vectors enter target cells and the genomic material is transcribed and translated for in vivo antigen production (Choi, Y. and J. Chang (2013). They can, but do not always, possess the ability to replicate within the host. Replication deficient vaccines may impart better safety, but immune memory is not as long-lasting. Currently, there are no viral vector vaccines used in the clinic for humans, but there are some that have been utilized for veterinary applications. 


\section{Preventive vaccine strategy}

Applied Nonotechnology

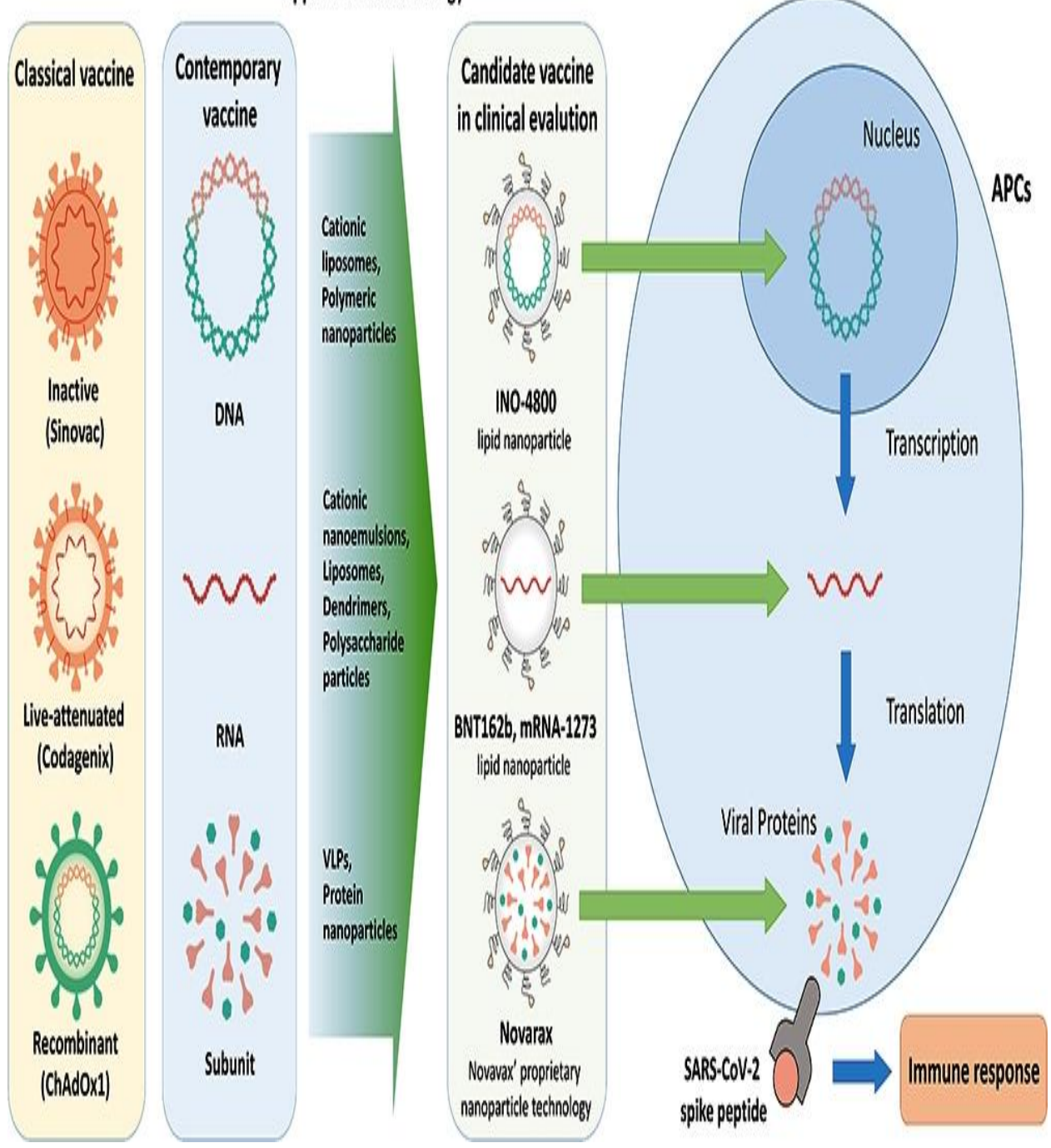


Figure (1) Classical vaccine, modern vaccine and nanotechnology applied vaccine against SARS-CoV-2. Types of classic vaccines and representative candidate vaccines in clinical trials, Nanoparticles applicable to contemporary vaccines using DNA, RNA, and subunits, representative candidate vaccines in clinical trials, and mechanism of action of nanotechnology-based vaccines in APC..Yang, D. (2021).

a)

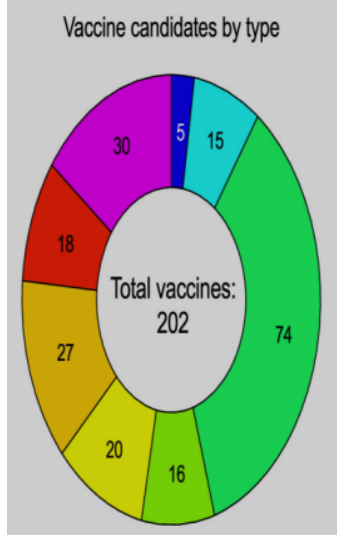

b)

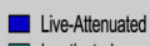

$\square$ Inactivated

$\square$ Subunit

$\square$ VLP

$\square$ Replicating Viral Vector

$\square$ Non-Replicating Viral Vector

$\square$ DNA

$\square$ RNA

c)

Vaccine candidates in clinical trials

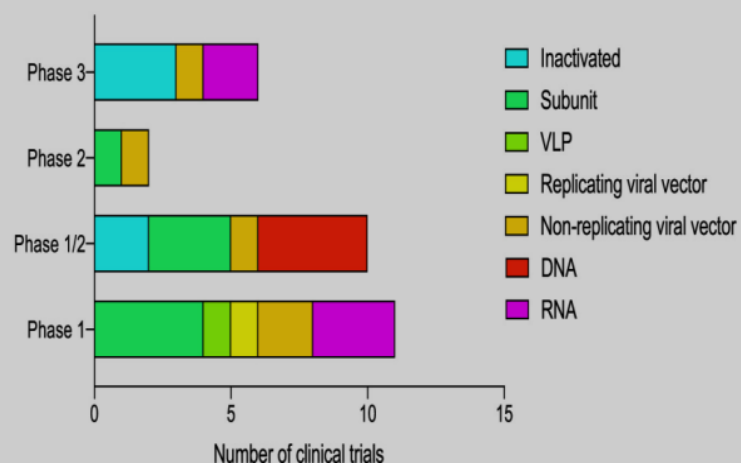

Usage of adiuvant

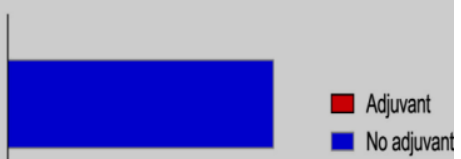

Number of vaccine candidates

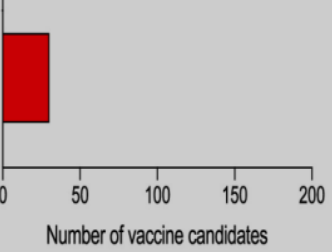

No adjuvant 
Figure 2. Graphs detailing the vaccines currently in development for SARS-CoV-2 according to the WHO and the Milken Institute as of August 11, 2020

a) Pie chart of the vaccines by type,

b) bar graph showing the number of vaccines using adjuvants,

c) Bar graph of the vaccine candidates in clinical trials. Hung, et.al,(2020).

The role of nanotechnology is highly relevant to counter this "virus" nano enemy. Nano intervention is discussed in terms of designing effective nanocarriers to counter the conventional limitations of antiviral and biological therapeutics. This strategy directs the safe and effective delivery of available therapeutic options using, blocking the initial interactions of viral spike glycoprotein with host cell surface receptors, and disruption of virion construction. Controlling and eliminating the spread and reoccurrence of this pandemic demands a safe and effective vaccine strategy. Nanocarriers have potential to design risk-free and effective immunization strategies for severe acute respiratory syndrome coronavirus 2 vaccine candidates such as protein constructs and nucleic acids figure 3 . The ongoing nanotechnology-based therapeutic and prophylactic strategies to fight against this pandemic, outlining the key areas for nanoscientists to step in Park, et.al, (2020). 


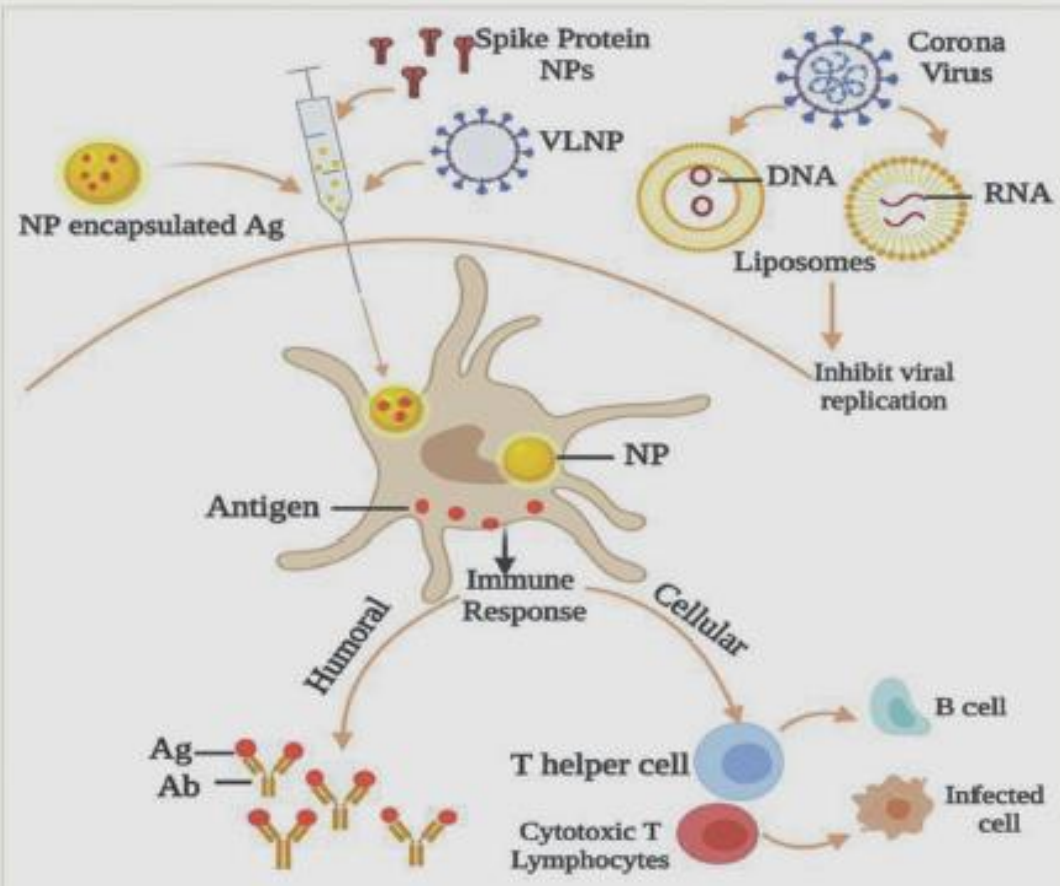

Figure (3) Nano particles (NP) antigen delivery system and immune response (Raiet.al, 2020)

Nanotechnology gives Opportunities in Vaccine Design

Nanoparticles and viruses operate at the same size scale; therefore, nanoparticles have an ability to enter cells to enable expression of antigens from delivered nucleic acids (mRNA and DNA vaccines) and/or directly target immune cells for delivery of antigens (subunit vaccines). Many vaccine technologies employ these direct benefits by encapsulating genomic material or protein/peptide antigens in nanoparticles such as lipid nanoparticles (LNPs) or other viruses such as Ads. BioNTech/Pfizer and Moderna encapsulate their mRNA vaccines within LNPs while the University of xford / AstraZeneca (from here on out referred to as Oxford/AstraZeneca) and CanSino incorporate antigen-encoding 
sequences within the DNA carried by Ads.Novavax decorates recombinant S proteins of SARS-CoV-2 onto their proprietary virus like particle (VLP) nanoparticles. Mulligan, et.al, 2020 and Zhu, et.al, (2020).

Moderna and BioNTech/Pfizer utilize mRNA vaccines that encode for the S protein of SARS-CoV-2. The $\mathrm{S}$ protein is the viral protein that binds to ACE2 on cells to mediate infection and is a frequent vaccine target since it is expected that antibodies binding to the correct epitope on the $S$ protein could be neutralizing and therefore block intercellular viral spread. The $\mathrm{S}$ protein has two subsections: S1 and S2. The S1 subunit contains the RBD and is responsible for initial attachment to the host cell through the ACE2 receptor, while the S2 subunit promotes viral fusion with cells to initiate infection. Moderna's vaccine, mRNA-1273, was codeveloped with the National Institute of Allergy and Infectious Diseases and specifically encodes the prefusion form of the S antigen (named S-2P) that includes a transmembrane anchor and an intact $\mathrm{S} 1-\mathrm{S} 2$ cleavage site. Jackson, et.al,(2020). Two proline substitutions in the vaccine mRNA at amino acids 986 and 987, which are within the central helix of the S2 subunit, keep the protein stable in its prefusion conformation. Wrapp, et.al.,(2020).

The mRNA is encapsulated within an LNP composed of four lipid. The exact formulation is not provided; however, inferences can be made based on previous LNP vaccines by Moderna, which utilize formulations of ionizable lipid, 1,2distearoyl-sn-glycero-3-phosphocholine, cholesterol, and polyethylene glycol-lipid. The exact lipids used are not stated. The mRNA-containing LNPs are solubilized and injected directly into the deltoid muscle. Moderna does not explicitly state the use of an adjuvant, but the LNP carrier may be an 
adjuvant since other lipids have been reported to have adjuvant properties Perrie, et.al,. (2016) and Richner, , et.al,. (2017). mRNA vaccine delivery systems applicable for COVID-19 vaccine progress.

To develop an effective LNP vector for delivery of COVID-19 mRNA vaccine, two critical factors should be taken into consideration as in Fig. (4) The first factor is choosing an appropriate cationic or ionizable lipid Thomas, K. (2020).Even though both cationic and ionizable lipids could effectively complex RNA; ionizable lipids may be preferred given their favorable safety profiles. Ionizable lipids are a class of lipid bearing neutral or mild positive charge at physiological $\mathrm{pH}$ and expose high cationic groups at acidic conditions. In this setting, the mRNA encoding SARS-CoV-2 antigens could be encapsulated into LNPs at low $\mathrm{pH}$, and LNPs could maintain a neutral surface charge in the extracellular space to reduce nonspecific interactions. Once LNPs are internalized into cells, the acidic environment within endosomes could turn the surface charge of LNPs to positive, facilitating endosomal escape and mRNA release in the cytosol. As the critical component of LNPs, great efforts have been devoted to identify optimal ionizable lipids Klein,et.al,. (2010) Representative ionizable lipids which were identified by high throughout screenings of large combinatorial libraries as Moderna (L5), which are biodegradable. Given the state of pandemic emergency, repurposing these high-performer ionizable lipids for a COVID19 mRNA vaccine formulation would be a good option. The second factor is optimizing cholesterol, helper phospholipids, and the lipid-PEG composition along with their relative ratio in LNPs. These factors could greatly affect the efficacy and performance of mRNA vaccines. However, the optimization process for formulations usually involves many more variables and requires extensive resources. To make the optimization 
process more efficient, Design of Experiment (DOE) methodologies have been applied, including both Fractional Factorial Designs and Definitive Screening Thomas, K. (2020). Through these methods, multiple parameters could be tuned simultaneously, such as the lipid ratio and lipid structure, leading them to find that incorporating DOPE and increasing ionizable lipid: mRNA ratio could increase the efficacy of mRNA delivery. Based on this, C12-200-containing LNP was optimized for erythropoietin mRNA delivery and a 7-fold potency improvement was observed. Similar approaches could also be applied for optimizing LNP for COVID-19 vaccine delivery. 

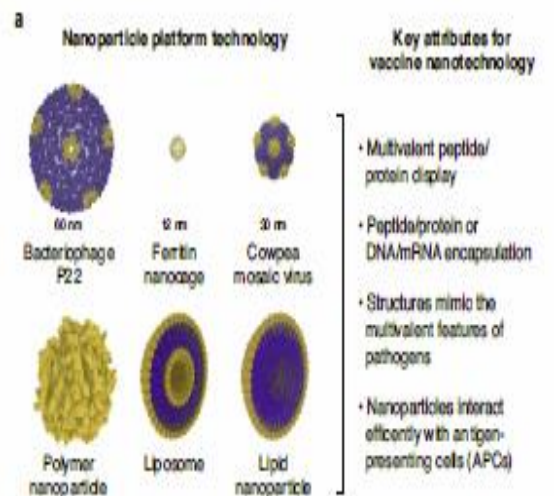

b

Nenoparticlobseod vaccine formulatons

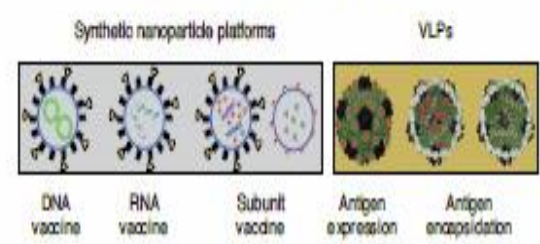

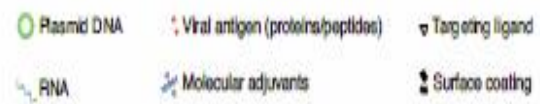

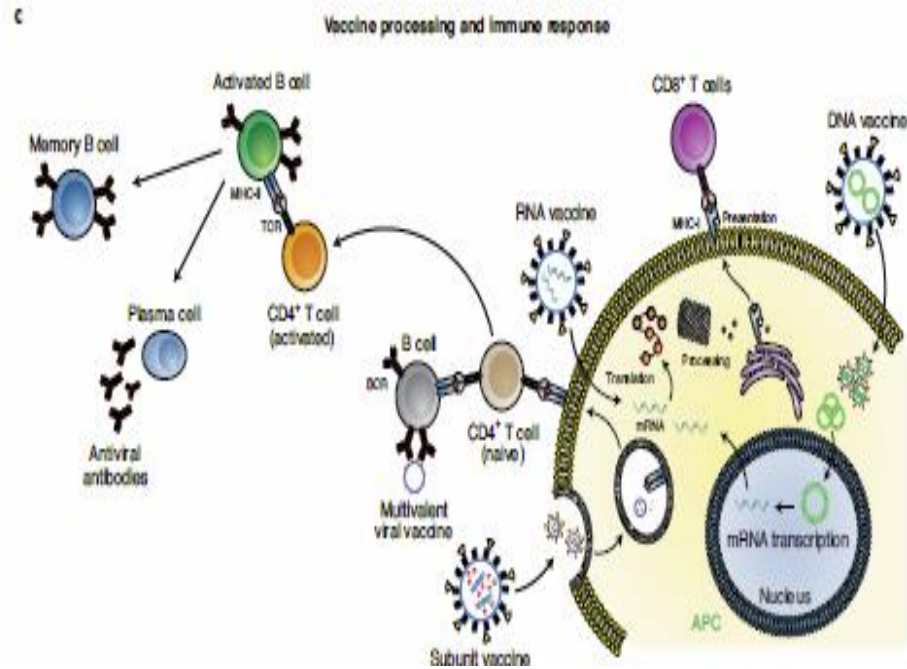

Fig. 4 | Nanoparticle platform vaccine technologies. a, Protein nanoparticles and their size; sizes for the synthetic nanocarriers vary between 10-1000 $\mathrm{nm}$. The protein nanoparticles were prepared using Chimera software using the PDB files (3IYI, 1FHA, $1 \mathrm{NY7}$ for P22, ferritin and CPMV, respectively). b, Components of nanoparticle-based vaccines. c, Key steps involved in nanoparticles-based vaccine processing by APCs. The antigenic cargo is processed by the APC and epitopes are 
presented by MHC-I and MHC-II leading to production of CD8+ cytotoxic $\mathrm{T}$ cells or $\mathrm{CD} 4+\mathrm{T}$ helper cells required for antiviral antibody production (or a combination thereof).Shin, et.al., (2020).

Apart from LNPs, cationic liposomes could also be used for mRNA-based vaccine applications, such as widely employed transfection agent, lipofectamine composed of (N-(1-(2,3dioleyloxy)propyl)-N-(2-(sperminecarboxamido)ethyl)-N,Ndimethylammonium trifluoroacetate (DOSPA) and (DOPE) Boyaka, P. N. (2017).

The optimized mRNA delivery system activated APCs and encoded various cancer antigens to induce strong antigenspecific T-cell responses, leading to tumor regression. These advances would allow for rapid design and development of ready-to-use mRNA vaccines targeting new emerging pathogens. Another important category of mRNA delivery system is based on polymer and polymer/lipid hybrid particles. Even though these delivery systems are not as clinically advanced as the lipidbased systems, their functional diversity and flexibility of polymers make them a very attractive candidate for mRNA delivery. For example, a PEI-based polyplex system has been used to deliver self-amplifying replicon RNA (RepRNA) vaccines encoding influenza virus hemagglutinin and nucleocapsid Zhang, et.al,(2020)

Other delivery systems, such as micelles and emulsions, have been used for mRNA delivery applications. Emulsions are well-established as potent vaccine adjuvants. Overall, mRNA vaccine is a powerful technology to fight against a pandemic outbreak, such as COVID-19 


\section{Pfizer-BioNTech COVID-19 Vaccine Suspension for Intramuscular Injection}

\section{Multiple Dose Vials}

(after dilution each vial contains

5 doses of $0.3 \mathrm{~mL}$ )

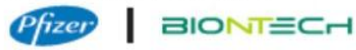

INGREDIENTS AND APPEARANCE

\section{PFIZER-BIONTECH COVID-19 VACCINE \\ rna ingredient bnt-162b2 injection, suspension}

\begin{tabular}{|c|c|c|c|c|}
\hline Product Type & $\begin{array}{l}\text { HUMAN PRESCRIPTION } \\
\text { DRUG }\end{array}$ & $\begin{array}{l}\text { Item Code } \\
\text { (Source) }\end{array}$ & & $\begin{array}{l}\text { NDC:59267- } \\
1000\end{array}$ \\
\hline $\begin{array}{l}\text { Route of } \\
\text { Administration }\end{array}$ & INTRAMUSCULAR & & \multirow{2}{*}{\multicolumn{2}{|c|}{$\begin{array}{c}\text { RNA code that instructs } \\
\text { cells to produce SARS CoV2 } \\
\text { Spike Protein }\end{array}$}} \\
\hline \multicolumn{3}{|c|}{ ACTIVE INGREDIENT/ACTIVE MOIETY } & & \\
\hline \multicolumn{2}{|c|}{ Ingredient Name } & \multicolumn{2}{|c|}{ Basis of Strength } & Strength \\
\hline \multicolumn{2}{|c|}{$\begin{array}{l}\text { RNA ingredient BNT-162B2 (UNII: 5085ZFP6S) (RNA } \\
\text { ingredient BNT-162B2 - UNII:5085ZFP6S) }\end{array}$} & \multicolumn{2}{|c|}{$\begin{array}{l}\text { RNA ingredient } \\
\text { BNT-162B2 }\end{array}$} & $\begin{array}{l}0.23 \mathrm{~g} \\
\text { in } 1.8 \mathrm{~mL}\end{array}$ \\
\hline \multicolumn{5}{|c|}{ INACTIVE INGREDIENTS } \\
\hline \multicolumn{3}{|c|}{ Ingredient Name } & \multirow{3}{*}{\multicolumn{2}{|c|}{$\begin{array}{c}\text { Strenath } \\
\text { Lipid nanoparticles that } \\
\text { carry and protect the mRNA } \\
\text { from degradation }\end{array}$}} \\
\hline \multicolumn{3}{|c|}{ lipid ALC-0159 (UNII: PJH39UMU6H) } & & \\
\hline \multicolumn{2}{|c|}{ lipid ALC-0315 (UNII: AVX8DX713V) } & & & \\
\hline \multicolumn{2}{|c|}{ POTASSIUM CHLORIDE (UNII: 660YQ98I10) } & \multicolumn{3}{|c|}{$\begin{array}{l}\text { Adjuvant used to antagonise the immune } \\
\text { system into a sustained response }\end{array}$} \\
\hline \multicolumn{4}{|c|}{ MONOBASIC POTASSIUM PHOSPHATE (UNII: 4J9FJOHL51) } & \multirow{3}{*}{$\begin{array}{l}\text { Ionic compounds } \\
\text { to balance } \mathrm{pH}\end{array}$} \\
\hline \multicolumn{4}{|c|}{ SODIUM CHLORIDE (UNII: 451W47IQ8X) } & \\
\hline \multicolumn{4}{|c|}{ SODIUM PHOSPHATE, DIBASIC, UNSPECIFIED FORM (UNII: GR686LBA74) } & \\
\hline \multicolumn{2}{|c|}{ SUCROSE (UNII: C151H8M554) } & prevent ins & redient s & eperation \& sticking \\
\hline
\end{tabular}

Figure $\square \square$. Scheme indicating the mechanism of mRNA-based antibody treatment. Reproduced with permission 


\section{References}

Boyaka, P. N. (2017). "Inducing mucosal IgA: a challenge for vaccine adjuvants and delivery systems." The Journal of Immunology 199(1): 9-16.

Bull, J. J., S. L. Nuismer and R. Antia (2019). "Recombinant vector vaccine evolution." PLoS computational biology 15(7): e1006857.

Campos, E. V., A. E. Pereira, J. L. De Oliveira, L. B. Carvalho, M. Guilger-Casagrande, R. De Lima and L. F. Fraceto (2020). "How can nanotechnology help to combat COVID-19? Opportunities and urgent need." Journal of Nanobiotechnology 18(1): 1-23.

Chauhan, G., M. J. Madou, S. Kalra, V. Chopra, D. Ghosh and S. O. Martinez-Chapa (2020). "Nanotechnology for COVID-19: therapeutics and vaccine research." ACS nano 14(7): 7760-7782.

Chen, W., U. Strych, P. Hotez and M. Bottazzi "March 2020." The SARS-CoV-2 vaccine pipeline: an overview. Curr Trop Med Rep https://doi. org/10.1007/s40475-02000201-6.

Choi, Y. and J. Chang (2013). "Viral vectors for vaccine applications." Clinical and experimental vaccine research 2(2): 97.

Chung, Y. H., V. Beiss, S. N. Fiering and N. F. Steinmetz (2020). "COVID-19 vaccine frontrunners and their nanotechnology design." ACS nano 14(10): 12522-12537.

Echevarría-Zuno, S., J. M. Mejía-Aranguré, A. J. MarObeso, C. Grajales-Muñiz, E. Robles-Pérez, M. González-León, M. C. Ortega-Alvarez, C. GonzalezBonilla, R. A. Rascón-Pacheco and V. H. Borja-Aburto (2009). "Infection and death from influenza A H1N1 virus in 
Mexico: a retrospective analysis." The Lancet 374(9707): 2072-2079.

Flotte, T. (2004). "Gene therapy progress and prospects: recombinant adeno-associated virus (rAAV) vectors." Gene therapy 11(10): 805-810.

Frey, S. E., P. L. Winokur, R. A. Salata, S. S. El-Kamary, C. B. Turley, E. B. Walter Jr, C. M. Hay, F. K. Newman, H. R. Hill and Y. Zhang (2013). "Safety and immunogenicity of IMVAMUNE® smallpox vaccine using different strategies for a post event scenario." Vaccine 31(29): 30253033.

Jackson, L. A., E. J. Anderson, N. G. Rouphael, P. C. Roberts, M. Makhene, R. N. Coler, M. P. McCullough, J. D. Chappell, M. R. Denison and L. J. Stevens (2020). "An mRNA vaccine against SARS-CoV-2-preliminary report." New England Journal of Medicine.

Klein, S. L., A. Jedlicka and A. Pekosz (2010). "The Xs and Y of immune responses to viral vaccines." The Lancet infectious diseases 10(5): 338-349.

Li, H., N. Malani and S. Hamilton (2011). "Assessing the potential for AAV vector genotoxicity in a murine model. Blood. 2011; 117 (12): 3311-3319." Blood 117(24).

Park, K. S., X. Sun, M. E. Aikins and J. J. Moon (2020). "Nonviral COVID-19 vaccine delivery systems." Advanced drug delivery reviews.

Mulligan, M., K. Lyke, N. Kitchin, J. Absalon, A. Gurtman, S. Lockhart, K. Neuzil, V. Raabe, R. Bailey and K. Swanson Phase 1/2 Study to Describe the Safety and Immunogenicity of a COVID-19 RNA Vaccine Candidate (BNT162b1) in Adults 18 to 55 Years of Age: Interim Report. medRxiv. 20202020.06. 30.20142570. 
Park, K. S., X. Sun, M. E. Aikins and J. J. Moon (2020). "Non-viral COVID-19 vaccine delivery systems." Advanced drug delivery reviews.

Perrie, Y., F. Crofts, A. Devitt, H. R. Griffiths, E. Kastner and V. Nadella (2016). "Designing liposomal adjuvants for the next generation of vaccines." Advanced drug delivery reviews 99: 85-96.

Pene, F., A. Merlat, A. Vabret, F. Rozenberg, A. Buzyn, F. Dreyfus, A. Cariou, F. Freymuth and P. Lebon (2003). "Coronavirus 229E-related pneumonia in immunocompromised patients." Clinical infectious diseases 37(7): 929-932.

Rai, M., S. Bonde, A. Yadav, Y. Plekhanova, A. Reshetilov, I. Gupta, P. Golińska, R. Pandit and A. P. Ingle (2020). "Nanotechnology-based promising strategies for the management of COVID-19: current development and constraints." Expert Review of Anti-infective Therapy: 1-10.

Richner, J. M., S. Himansu, K. A. Dowd, S. L. Butler, V. Salazar, J. M. Fox, J. G. Julander, W. W. Tang, S. Shresta and T. C. Pierson (2017). "Modified mRNA vaccines protect against Zika virus infection." Cell 168(6): 1114-1125. e1110.

Ruiz-Hitzky, E., M. Darder, B. Wicklein, C. Ruiz-Garcia, R. Martín-Sampedro, G. Del Real and P. Aranda (2020). "Nanotechnology responses to COVID-19." Advanced healthcare materials 9(19): 2000979.

Shin, M. D., S. Shukla, Y. H. Chung, V. Beiss, S. K. Chan, O. A. Ortega-Rivera, D. M. Wirth, A. Chen, M. Sack and J. K. Pokorski (2020). "COVID-19 vaccine development and a potential nanomaterial path forward." Nature nanotechnology 15(8): 646-655. 
Sicot, G., G. Gourdon and M. Gomes-Pereira (2011). "Myotonic dystrophy, when simple repeats reveal complex pathogenic entities: new findings and future challenges." Human Molecular Genetics 20(R2): R116-R123.

Thao, T. T. N., F. Labroussaa, N. Ebert, P. V'kovski, H. Stalder, J. Portmann, J. Kelly, S. Steiner, M. Holwerda and A. Kratzel (2020). "Rapid reconstruction of SARSCoV-2 using a synthetic genomics platform." Nature 582(7813): 561-565.

Thomas, K. (2020). "US Will Pay \$1.6 Billion to Novavax for Coronavirus Vaccine." New York Times.

Vartak, A. and S. J. Sucheck (2016). "Recent advances in subunit vaccine carriers." Vaccines 4(2): 12.

Weiss, C., M. Carriere, L. Fusco, I. Capua, J. A. Regla-Nava, M. Pasquali, J. A. Scott, F. Vitale, M. A. Unal and C. Mattevi (2020). "Toward nanotechnology-enabled approaches against the COVID-19 pandemic." ACS nano 14(6): 6383-6406.

Wrapp, D., N. Wang, K. S. Corbett, J. A. Goldsmith, C.-L. Hsieh, O. Abiona, B. S. Graham and J. S. McLellan (2020). "Cryo-EM structure of the 2019-nCoV spike in the prefusion conformation." Science 367(6483): 1260-1263

Yang, D. (2021). "Application of Nanotechnology in the COVID-19 Pandemic." International Journal of Nanomedicine 16: 623.

Zhang, Y., X. Geng, Y. Tan, Q. Li, C. Xu, J. Xu, L. Hao, Z. Zeng, $X$. Luo and F. Liu (2020). "New understanding of the damage of SARS-CoV-2 infection outside the respiratory system." Biomedicine \& Pharmacotherapy: 110195.

Zhu, F.-C., Y.-H. Li, X.-H. Guan, L.-H. Hou, W.-J. Wang, J.X. Li, S.-P. Wu, B.-S. Wang, Z. Wang and L. Wang (2020). "Safety, tolerability, and immunogenicity of a recombinant adenovirus type-5 vectored COVID-19 vaccine: 
a dose-escalation, open-label, non-randomised, first-inhuman trial." The Lancet 395(10240): 1845-1854. 